CASE REPORT

\author{
C.-S. Tung \\ S.-L. Wu \\ J.-C. Tsou \\ S.-P. Hsu \\ H.-C. Kuo \\ H.-W. Tsui
}

\section{Marchiafava-Bignami Disease with Widespread Lesions and Complete Recovery}

SUMMARY: MBD is a rare disorder strongly associated with alcoholism. It is characterized pathologically by demyelination and necrosis of the corpus callosum. MBD presents with severe neurologic deficits and significant sequelae developing in most survivors. We report a patient with total clinical recovery. Serial MR imaging demonstrated typical lesions with restricted diffusion in the acute stage and total resolution without atrophy or cystic change.

ABBREVIATIONS: $A D C=$ apparent diffusion coefficient; DWI = diffusion-weighted imaging; $\mathrm{MBD}=$ Marchiafava-Bignami disease $; \mathrm{T} 1 \mathrm{WI}=\mathrm{T} 1$-weighted imaging; $\mathrm{T} 2 \mathrm{WI}=\mathrm{T} 2$-weighted imaging
M $\mathrm{BD}$ is a rare disorder in individuals with chronic alcoholism and is characterized by severe neurologic symptoms and symmetric lesions in the corpus callosum. Cases with a favorable outcome have been reported in recent years. ${ }^{1,2}$ Nevertheless, most patients with MBD who survive have had significant sequelae. We present a patient with MBD showing complete resolution of the MR imaging abnormalities and clinical recovery, despite severe initial presentation and widespread lesions.

\section{Case Report}

A 48-year-old man with a history of type II diabetes mellitus and chronic excessive alcohol consumption for $>20$ years had vertigo, slurred speech, and progressive gait disturbance. He was referred to us due to a rapidly deteriorating level of consciousness 6 days later. On admission, he was comatose, and findings of neurologic examinations showed tetraparesis, with generalized muscular hypertonia. There was mild hyperglycemia and hypokalemia. Blood test results, including biochemistry, electrolytes, nutrition, and endocrine function, were all within normal limits. Tests of urinary amphetamine and morphine levels were negative. The CSF analysis showed isolated mild hyperproteinorachia.

Findings of brain CT performed 3 days after onset were normal. MR imaging on day 5 (Fig 1) demonstrated hyperintensity at the corpus callosum, hemispheric white matter, internal capsules, cerebral peduncles, and middle cerebellar peduncles on T2WI and DWI. The lesion in the corpus callosum appeared hypointense on T1WI. No abnormal enhancement was noted after gadolinium injection.

On the basis of clinical and imaging features, MBD was diagnosed. Intravenous vitamin B complex and methylprednisolone therapy ( $1000 \mathrm{mg} /$ day for 3 days) were started on day 7 . On day 17 , he regained normal attention, and a follow-up MR imaging demonstrated marked resolution of the lesions in the hemispheric white matter and middle cerebellar peduncles. There were residual hyperintense lesions at the splenium of the corpus callosum, internal capsules, and cerebral peduncles on T2WI and DWI. ADC mapping showed relative hypointensity in the aforementioned lesions (Fig $2 A-C$ ).

Approximately 4 weeks after the onset, he had a total recovery.

Received August 25, 2009; accepted after revision August 26

From the Department of Neurology, E-Da Hospital, I-Shou University, Kaohsiung County, Taiwan, R.O.C

Please address correspondence to Huan-Wen Tsui, MD, Department of Neurology, E-Da Hospital, I-Shou University, 1 E-Da Rd, Jiau-Shu Tsuen, Yan-Chau Shiang, Kaohsiung County, Taiwan, 824, R.0.C.; e-mail: ed100879@edah.org.tw

DOI 10.3174/ajnr.A1897
Follow-up MR imaging performed 3 months after onset showed disappearance of signal-intensity abnormalities on T1WI, T2WI, and DWI (Fig $2 D-F$ ), without atrophy or cystic change of the corpus callosum. Four months later, he returned to his prior occupation as a construction worker without difficulty. He continued total abstention of alcoholic consumption.

\section{Discussion}

The presentations of $\mathrm{MBD}$ are variable. In the acute stage, the patient often has nonspecific neurologic changes, such as confusion, coma, seizure, dysarthria, and hemiparesis. ${ }^{2,3}$ Classic interhemispheric disconnection syndrome is often obscure in patients with confusional state, and it has often been misdiagnosed as Wernicke encephalopathy, alcohol withdrawal syndrome, or encephalitis. The early diagnosis depends on MR imaging. Conditions with acute onset, impairment of consciousness, widespread lesions, and cortical involvement indicate an unfavorable prognosis. ${ }^{2,3}$

The characteristic MR imaging findings are symmetric lesions of the corpus callosum. Lesions may be also found in the hemispheric white matter, cortex, middle cerebellar peduncles, and internal capsules. ${ }^{3,4}$ During the acute phase, the affected areas demonstrate edematous change with or without demyelination, appearing hypointense on T1WI and hyperintense on T2WI. After the acute stage, edematous change may subside and the corpus callosum gradually recovers its normal intensity. In the case of permanent myelin damage and necrosis, MR imaging typically reveals atrophy of the corpus callosum with cystic change, particularly in the central layer.

In our case, the rapid resolution of the widespread callosal and extracallosal hyperintensities on DWI was highly unusual. The DWI and ADC values on day 17 showed restriction of water diffusion in the splenium. This finding is also seen in acute infarction due to cytotoxic edema, and it always has irreversible change, contrary to the rapid resolution in MBD. We proposed that this phenomenon may be caused by reversible myelin vacuolization or intramyelinic edema, which has been described in patients with epilepsy and mild encephalitis. $^{5,6}$

CT is not a sensitive tool for the detection of early lesions; MR imaging is more useful for early diagnosis and follow-up in MBD. In our case, presenting with major impairment of consciousness and widespread lesions is not necessarily linked to an ominous outcome. A diminution of ADC in the acute stage may not be correlated with irreversibility. There may be 

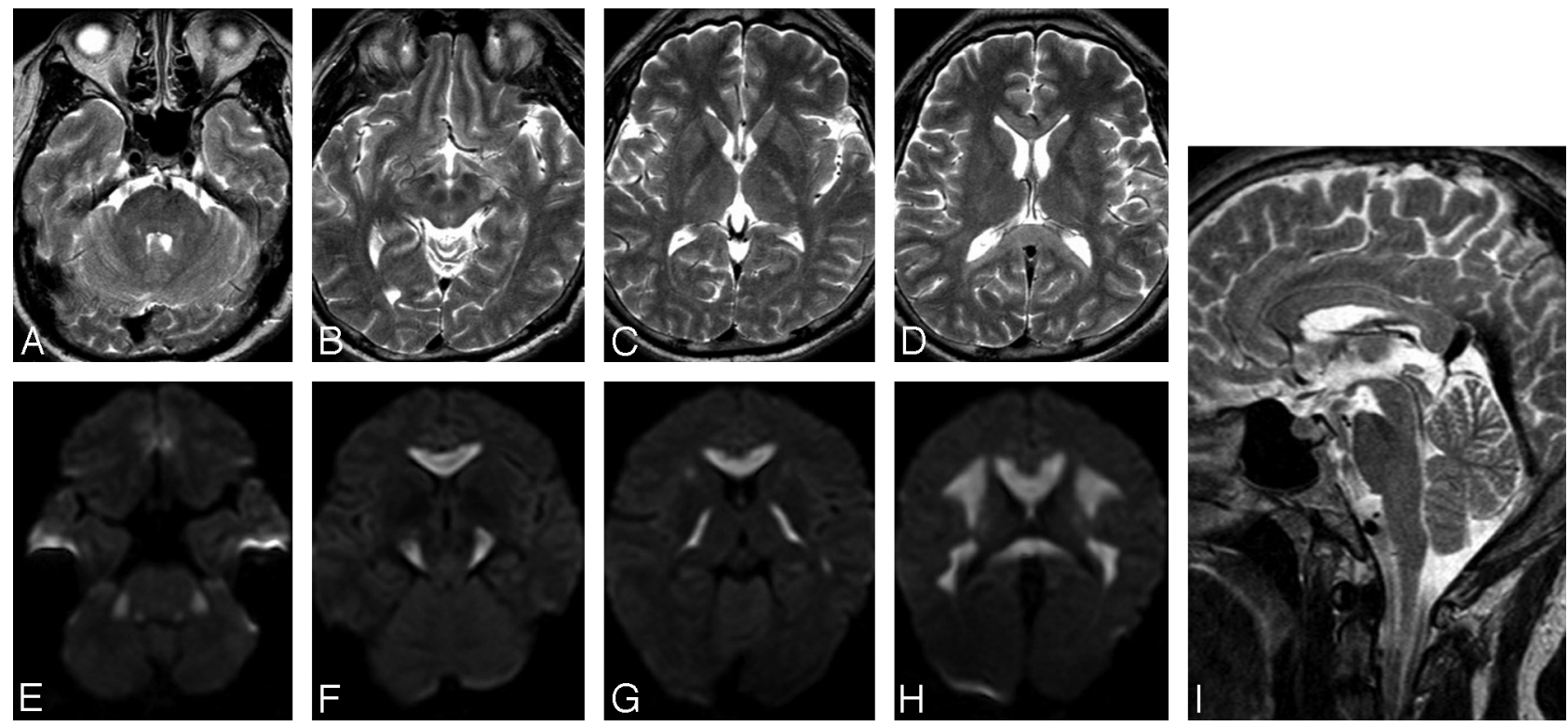

Fig 1. MR images obtained on day 5. $A-D$, On axial T2WI, the lesions appear hyperintense in the middle cerebellar peduncles, cerebral peduncles, internal capsules, and hemispheric white matter. E-H, On corresponding DWI, the lesions appear hyperintense. I, On sagittal T2WI, hyperintense lesions are seen throughout the corpus callosum.
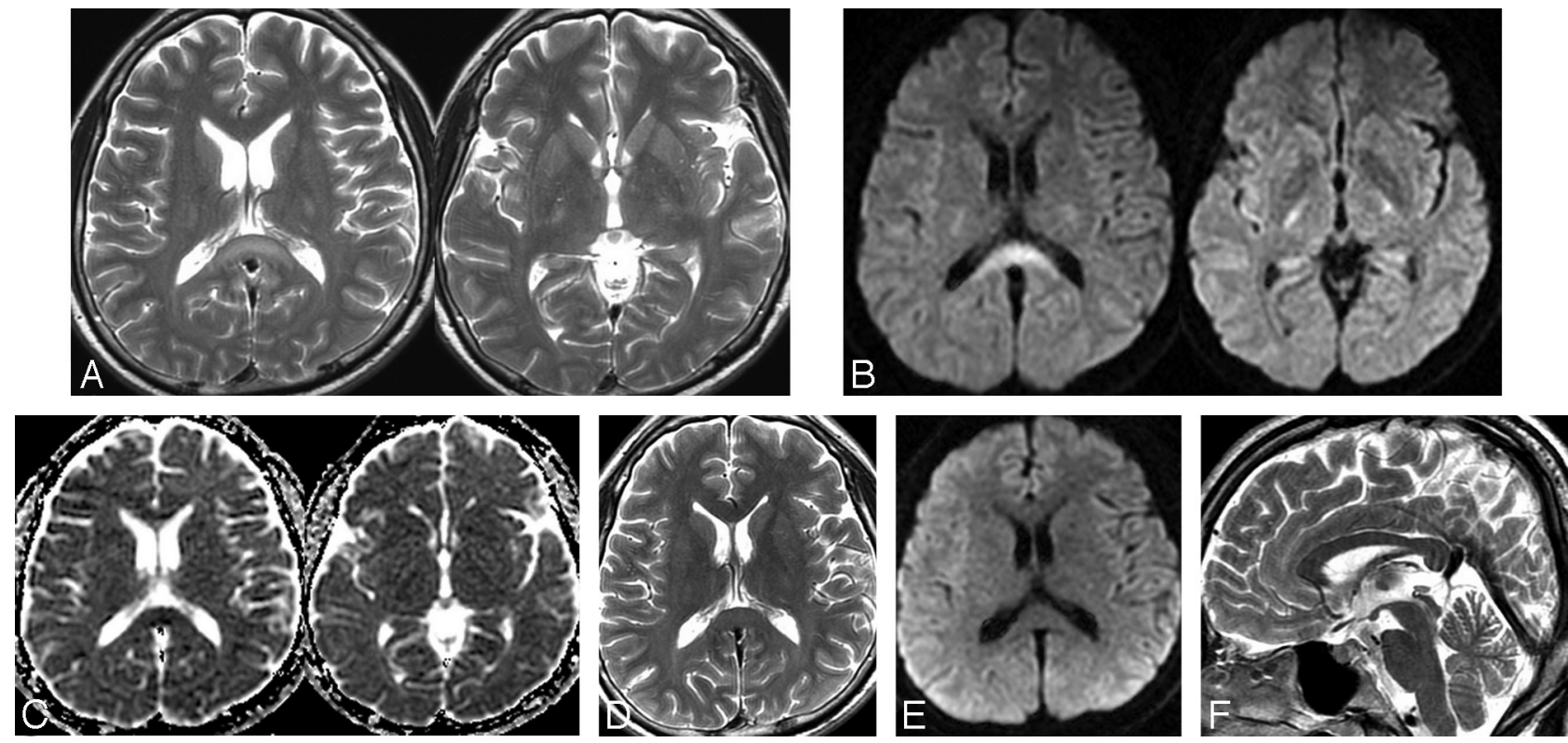

Fig 2. Follow-up $M R$ images obtained on days $17(A-C)$ and $30(D-F)$. $A$, On axial T2WI, hyperintense lesions are seen in the splenium and internal capsules. $B$ and $C$, On axial $D W$ and an ADC map, the lesions appear hyperintense in DWI and hypointense on the ADC map in the splenium and internal capsule. $D-F$, On follow-up MR images obtained 3 months after onset, note the disappearance of all signal-intensity abnormalities on axial T2WI, DWI, and sagittal T2WI.

a therapeutic window in which early diagnosis and treatment may improve the prognosis.

\section{References}

1. Ruiz-Martinez J, Martinez Perez-Balsa A, Ruibal M, et al. Marchiafava-Bignami disease with widespread extracallosal lesions and favourable course. Neuroradiology 1999;41:40-43

2. Heinrich A, Runge U, Khaw AV. Clinicoradiologic subtypes of MarchiafavaBignami disease. J Neurol 2004;251:1050-59
3. Menegon P, Sibon I, Pachai C, et al. Marchiafava-Bignami disease: diffusionweighted MRI in corpus callosum and cortical lesions. Neurology 2005;65:475-77

4. Kohler CG, Ances BM, Coleman AR, et al. Marchiafava-Bignami disease: literature review and case report. Neuropsychiatry Neuropsychol Behav Neurol 2000;13:67-76

5. Oster J, Doherty C, Grant PE, et al. Diffusion-weighted imaging abnormalities in the splenium after seizures. Epilepsia 2003;44:852-54

6. Tada H, Takanashi J, Barkovich AJ, et al. Clinically mild encephalitis/encephalopathy with a reversible splenial lesion. Neurology 2004;63:1854-58 